Gut and Liver, Vol. 12, No. 5, September 2018, pp. 571-582

\title{
Assessment of the Surveillance Interval at 1 Year after Curative Treatment in Hepatocellular Carcinoma: Risk Stratification
}

\author{
Minjong Lee ${ }^{1,2}$, Young Chang ${ }^{1}$, Sohee $\mathrm{Oh}^{3}$, Young Youn $\mathrm{Cho}^{1}$, Dhong-Eun Jung ${ }^{1}$, Hong Hyun $\mathrm{Kim}^{1}$, Joon Yeul Nam ${ }^{1}$, \\ Hyeki Cho ${ }^{1}$, Eun Ju Cho ${ }^{1}$, Jeong-Hoon Lee ${ }^{1}$, Su Jong Yu ${ }^{1}$, Nam-Joon $\mathrm{Yi}^{4}$, Kwang-Woong Lee ${ }^{4}$, Dong Ho Lee ${ }^{5}$, Jeong Min \\ Lee $^{5}$, Jung-Hwan Yoon ${ }^{1}$, Kyung-Suk Suh ${ }^{4}$, and Yoon Jun Kim ${ }^{1}$
}

${ }^{1}$ Department of Internal Medicine and Liver Research Institute, Seoul National University College of Medicine, Seoul, ${ }^{2}$ Department of Internal Medicine, Kangwon National University Hospital, Chuncheon, ${ }^{3}$ Department of Biostatistics, Seoul Metropolitan Government Seoul National University Boramae Medical Center, Departments of ${ }^{4}$ Surgery and ${ }^{5}$ Radiology, Seoul National University College of Medicine, Seoul, Korea

Background/Aims: Guidelines recommend surveillance for hepatocellular carcinoma (HCC) recurrence at 3-month intervals during the first year after curative treatment and 6-month intervals thereafter in all patients. This strategy does not reflect individual risk of recurrence. We aimed to stratify risk of recurrence to optimize surveillance intervals 1 year after treatment. Methods: We retrospectively analyzed 1,316 HCC patients treated with resection/radiofrequency ablation at Barcelona Clinic Liver Cancer stage 0/ A. In patients without 1-year recurrence under 3-monthly surveillance, a new model for recurrence was developed using backward elimination methods: training $(n=582) /$ validation cohorts $(n=291)$. Overall survival (OS) according to risk stratified by the new model was compared according to surveillance intervals: 3-monthly versus 6-monthly $(n=401)$ after lead time bias correction and propensity-score matching analyses. Results: Among patients without 1-year recurrence, age and international normalized ratio values were significant factors for recurrence (hazard ratio [HR], 1.03; 95\% confidence interval [Cl], 1.00 to 1.03; $p=0.009$ and HR, 5.63; 95\% Cl, 2.24 to 14.18; $p<0.001$; respectively). High-risk patients stratified by the new model showed significantly higher recurrence rates than low-risk patients in the validation cohort ( $\mathrm{HR}, 1.73$; $95 \% \mathrm{Cl}, 1.18$ to 2.53 ; $\mathrm{p}=0.005$ ). After propensity-score matching between the 3-monthly and 6-monthly surveillance groups, OS in high-risk patients under 3-monthly surveillance was significantly higher than that under 6-monthly surveillance $(p=0.04)$; however, OS in low-risk patients under 3-monthly surveillance was not significantly different from that under 6-monthly surveillance $(p=0.17)$. Conclusions: In high-risk patients, 3-monthly surveillance can prolong survival compared to 6-monthly surveillance. However, in low-risk patients, 3-monthly surveillance might not be beneficial for survival compared to 6-monthly surveillance. (Gut Liver 2018;12:571-582)

Key Words: Surveillance interval; Carcinoma, hepatocellular; Curative treatment; Risk stratification

\section{INTRODUCTION}

The current distribution of hepatocellular carcinoma (HCC) based on the Barcelona Clinic Liver Cancer (BCLC) stage is approximately $30 \%$ to $40 \%$ of patients having early stage disease. Particularly in Japan, the proportion of early stage HCC is approximately 65\%, probably because of a very well-established surveillance system. ${ }^{1-3}$ Although surgery or radiofrequency ablation (RFA) is the mainstay of effective treatment for HCC in BCLC stages 0 or A, HCC recurs in 70\% of patients within 5 years and includes either intrahepatic metastases or the development of de novo tumors. ${ }^{4-8}$ Therefore, postoperative surveillance for early detection of recurrence is an important approach for curative therapies and long-term survival, particularly in early cirrhotic patients in BCLC stages 0/A. ${ }^{9-12}$

In the current guidelines suggested by the European Association for the Study of the Liver, follow-up strategies for detection of recurrence indicate contrast-enhanced computed tomography (CT) surveillance every 3 months during the first year, and every

Correspondence to: Yoon Jun Kim

Department of Internal Medicine and Liver Research Institute, Seoul National University College of Medicine, 101 Daehak-ro, Jongno-gu, Seoul 03080, Korea

Tel: +82-2-2072-3081, Fax: +82-2-743-6701, E-mail: yoonjun@snu.ac.kr

Received on August 7, 2017. Revised on January 30, 2018. Accepted on February 26, 2018. Published online June 22 , 2018

pISSN 1976-2283 eISSN 2005-1212 https://doi.org/10.5009/gnl17365

Minjong Lee and Young Chang contributed equally to this work as first authors.

@ This is an Open Access article distributed under the terms of the Creative Commons Attribution Non-Commercial License (http://creativecommons.org/licenses/by-nc/4.0) which permits unrestricted non-commercial use, distribution, and reproduction in any medium, provided the original work is properly cited. 
6 months thereafter. ${ }^{2}$ However, all of the guidelines did not reflect the individual risk considering patients' tumor biology and liver function for survival.

In this study, we aimed to identify patients who could have extended surveillance intervals at the first year after curative treatments in patients with HCC in BCLC stage 0/A and with well-preserved liver function.

\section{MATERIALS AND METHODS}

\section{Patients}

A retrospective cohort study was conducted with 1,318 consecutively registered patients who underwent surgical resection or RFA as the first curative treatment at a tertiary hospital (Seoul National University Hospital, Seoul, Korea) between January 2001 and June 2015. The inclusion criteria were as follows: (1) patients with HCC BCLC stages 0/A with a performance status score of 0; (2) Child-Turcotte-Pugh (CTP) score $\leq 7$; (3) successful treatment with resection (R0) or RFA (sufficient safety margin) as a first-line anti-tumor treatment; (4) recurrence diagnosed during a regular surveillance visit based on liver CT or magnetic resonance imaging (MRI) performed every 3 months or 6 months with or without alpha-fetoprotein (AFP) determination; and (5) completed visits on a regular basis according to each surveillance program until tumor recurrence was documented, and good compliance such as taking antiviral medications or abstaining from alcohol.

The following were grounds for exclusion: (1) patients who received the first treatments, except for resection or RFA, such as trans-arterial chemoembolization, or percutaneous ethanol injection; (2) CTP score >7; (3) BCLC B/C/D stage; (4) patients who suffered from recurred HCC at baseline; (5) patients who had combined HCC and cholangiocarcinoma, and diffuse infiltrative types of HCC; (6) patients who were treated with liver transplantation; (7) patients diagnosed with any other known malignancies; and (8) patients who exhibited poor compliance for antiviral therapy or alcohol abstinence (Fig. 1).

The study protocol complied with the ethical guidelines of the World Medical Association Declaration of Helsinki.

This study was approved by the institutional review board of Seoul National University Hospital (No. 1502-077-649), and the requirement for informed consent from patients was waived.

\section{Preoperative and postoperative assessment}

Treatment strategies for initial HCC such as resection and RFA were described in Supplementary Material 1. Evaluation was performed 1 month after curative treatment, and then 3 -monthly until year 1 . Since year 1 , there were two groups for surveillance: under 3-monthly and 6-monthly surveillance until tumor recurrence was documented. At every visit, imaging studies such as CT scan, and serologic tests such as tumor markers and biochemical liver function tests, were performed.

Regarding assignment of patients to 3- or 6-monthly surveillance after curative treatment, there might be bias of the physicians: physicians might prefer 6-monthly surveillance after treatment in patients with low-risk for overall survival (OS) and 3-monthly surveillance after treatment in patients with highrisk for OS due to retrospective design of this study. To minimize physicians' bias for patients' risk, risk stratification was performed in both groups by the developed model in this study and survival rates of same risk group were compared according to surveillance intervals such as 3 months or 6 months.

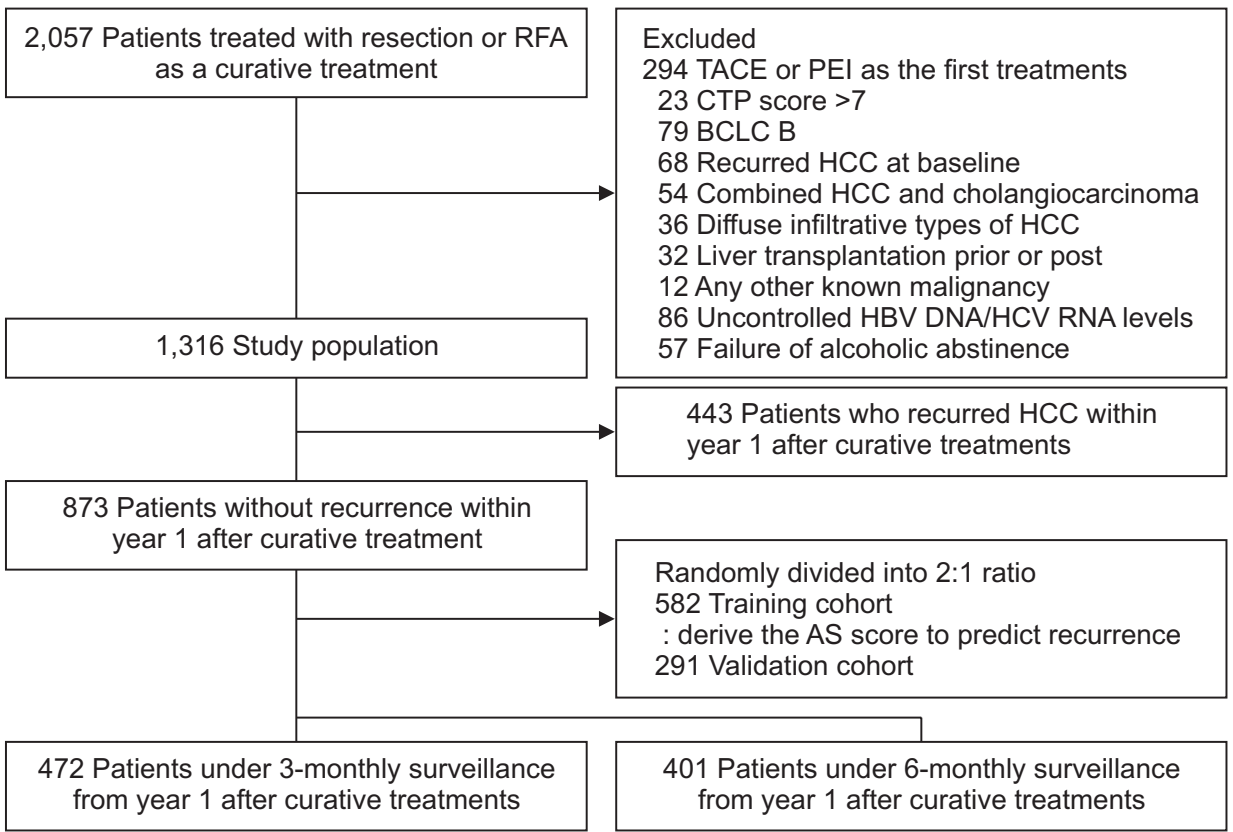

Fig. 1. Patients flow diagram. RFA, radio-frequency ablation; TACE, trans-arterial chemo-embolization; PEI, percutaneous ethanol injection; CTP, Child-Turcotte-Pugh; BCLC, Barcelona Clinic Liver Cancer; HCC, hepatocellular carcinoma; HBV, hepatitis B virus; HCV, hepatitis $C$ virus; AS score, assessment for surveillance interval score. 


\section{Definition}

Initial HCC was diagnosed according to the current guidelines (Supplementary Material 2). ${ }^{2,13}$ A new model to predict HCC recurrence and stratify the risk was developed. Based on risk of HCC recurrence stratified by a new model, OS between low-risk and high-risk groups was compared according to surveillance intervals such as 3 months or 6 months. OS was defined as the time from first diagnosis of HCC until death. Cirrhotic complications were included ascites, variceal bleeding, spontaneous bacterial peritonitis, hepatic encephalopathy, and hepatorenal syndrome. ${ }^{14}$

Total population was 1,316 patients comprised of 915 patients under 3-monthly surveillance and 401 patients under 6-monthly surveillance after curative treatment.

\section{Development of a new model to predict recurrence, and comparison of survival between 3-monthly and 6-monthly surveillance}

Multivariable analysis to identify risk factors predictive of OS

Table 1. Baseline Characteristics

\begin{tabular}{|c|c|c|c|c|}
\hline \multirow[b]{2}{*}{ Variable } & \multirow[b]{2}{*}{ Total population $(\mathrm{n}=1,316)$} & \multicolumn{3}{|c|}{ HCC patients without 1-yr recurrence } \\
\hline & & $\begin{array}{l}\text { Training cohort } \\
\quad(\mathrm{n}=582)\end{array}$ & $\begin{array}{l}\text { Validation cohort } \\
\qquad(\mathrm{n}=291)\end{array}$ & p-value* \\
\hline Age, yr & $58(52-65)$ & $58(52-65)$ & $58(51-65)$ & 0.56 \\
\hline Male sex & $988(75.1)$ & $431(74.1)$ & $214(73.5)$ & 0.93 \\
\hline Etiology & & & & 0.43 \\
\hline $\mathrm{HBV}$ & 967 (74.6) & 430 (73.9) & $224(77.0)$ & \\
\hline $\mathrm{HCV}$ & $131(10.1)$ & $50(8.6)$ & $28(9.6)$ & \\
\hline $\mathrm{HBV}+\mathrm{HCV}$ & $19(1.5)$ & $10(1.7)$ & $5(1.7)$ & \\
\hline Others $^{\dagger}$ & $179(13.8)$ & $92(15.8)$ & $34(11.7)$ & \\
\hline CTP score & $5(5-5)$ & $5(5-5)$ & $5(5-5)$ & 0.96 \\
\hline MELD score & $8(7-10)$ & $8(7-9)$ & $8(7-10)$ & 0.04 \\
\hline AST, U/L & $36(27-50)$ & $34(26-47)$ & $36(26-50)$ & 0.30 \\
\hline ALT, U/L & $35(24-50)$ & $35(24-50)$ & $34(24-50)$ & 0.85 \\
\hline Albumin, $\mathrm{g} / \mathrm{dL}$ & $4.1(3.8-4.3)$ & $4.1(3.8-4.3)$ & $4.1(3.8-4.3)$ & 0.58 \\
\hline Total bilirubin, mg/dL & $1.0(0.8-1.3)$ & $1.0(0.7-1.2)$ & $1.0(0.8-1.2)$ & 0.11 \\
\hline Creatinine, $\mathrm{mg} / \mathrm{dL}$ & $0.9(0.8-1.1)$ & $0.9(0.8-1.1)$ & $0.9(0.8-1.1)$ & 0.97 \\
\hline PT INR & $1.1(1.0-1.2)$ & $1.1(1.0-1.2)$ & $1.1(1.0-1.2)$ & 0.08 \\
\hline Platelet, $\times 10^{3} / \mu \mathrm{L}$ & 130 (94-170) & $137(100-174)$ & 131 (97-175) & 0.39 \\
\hline AFP, ng/mL & $11.3(5.0-94.3)$ & $10.2(4.9-76.0)$ & $9.8(4.0-56.7)$ & 0.26 \\
\hline Tumor size, $\mathrm{cm}$ & $2.3(1.7-3.0)$ & $2.3(1.6-3.0)$ & $2.1(1.6-3.0)$ & 0.94 \\
\hline No. of tumor & & & & 0.29 \\
\hline 1 & $1,145(87.0)$ & $517(88.8)$ & 259 (89.0) & \\
\hline 2 & 139 (10.6) & $57(9.8)$ & $24(8.2)$ & \\
\hline 3 & $32(2.4)$ & $8(1.4)$ & $8(2.8)$ & \\
\hline BCLC stage & & & & 0.71 \\
\hline 0 & 457 (34.7) & 217 (37.3) & 113 (38.8) & \\
\hline A & $859(65.3)$ & $365(62.7)$ & $178(61.2)$ & \\
\hline Treatment method & & & & 0.42 \\
\hline RFA & $812(61.7)$ & $356(61.2)$ & $169(58.1)$ & \\
\hline Resection & 504 (38.3) & 226 (38.8) & $122(41.9)$ & \\
\hline
\end{tabular}

Data are presented as median (interquartile range) or number (\%).

HCC, hepatocellular carcinoma; HBV, hepatitis B virus; HCV, hepatitis C virus; CTP, Child-Turcotte-Pugh; MELD, Model for End-Stage Liver Disease; AST, aspartate aminotransferase; ALT, alanine aminotransferase; PT INR, international normalized ratio for prothrombin time; AFP, alphafetoprotein; BCLC, Barcelona Clinic Liver Cancer; RFA, radiofrequency ablation.

*p-value estimated by the chi-square test or Fisher exact test for categorical variables and the Mann-Whitney U test for comparison between the training and validation cohorts; ${ }^{\dagger}$ Other causes included alcohol, non-alcoholic fatty liver disease, primary biliary cholangitis, and autoimmune hepatitis. 
was performed among the patients under 3-monthly surveillance in the total population ( $\mathrm{n}=915)$. To determine surveillance intervals at the time of 1 year in patients who did not develop HCC within 1 year after curative treatments, a new model for risk stratification for HCC recurrence was derived from patients without 1-year recurrence.

Patients $(n=873)$ who did not recur within 1 year after curative treatment were randomly divided into two groups with 2:1 ratio to derive and validate a new model for risk stratification at year 1 after treatment: training $(n=582)$ and validation cohorts $(\mathrm{n}=291)$. Survival rates in patients under 3-monthly surveillance $(\mathrm{n}=472)$ were compared to those under 6-monthly surveillance $(n=401)$. To assess the survival benefit of a 3-month surveillance interval rather than a 6-month interval from year 1 after treatment according to risk by the new model, we compared the survival rates between patients under 3-monthly surveillance $(\mathrm{n}=472)$ and those under 3-monthly surveillance for 1 year after treatment and thereafter 6-monthly interval surveillance using the propensity-score matching $(n=401)$.

\section{Statistical analyses}

To minimize the lead time bias, ${ }^{15}$ the "lead time" was calculated for a 3-month surveillance interval patients by Schwartz's formula, ${ }^{16}$ which was originally proposed for calculating tumor growth: $t=D T \times 3 \times \log (d 1 / d 0) / \log (2)$, where $t$ is the lead time (days), DT is the median value of the tumor volume doubling time proposed by Sheu et al., ${ }^{17}$ do is the median tumor diameter of patients under 3-monthly surveillance, and $\mathrm{d} 1$ is the median tumor diameter of patients under 6-monthly surveillance. The calculated lead time for patients examined every 3 months was subtracted from their survival and recurrence. If the value became negative, we attributed a survival (deceased patients) or

Table 2. Multivariable Analysis for Overall Mortality ( $\mathrm{n}=915)$

\begin{tabular}{|c|c|c|c|c|}
\hline \multirow{2}{*}{ Variable } & \multicolumn{2}{|c|}{ Univariable } & \multicolumn{2}{|c|}{ Multivariable } \\
\hline & HR $(95 \%$ CI) & p-value* & HR (95\% CI) & p-value ${ }^{*}$ \\
\hline Age, yr & $1.03(1.01-1.04)$ & $<0.001$ & $1.01(0.99-1.03)$ & 0.28 \\
\hline Male sex & $0.86(0.64-1.16)$ & 0.34 & & \\
\hline \multicolumn{5}{|l|}{ Etiology } \\
\hline $\mathrm{HCV}$ & $1.86(1.29-2.69)$ & $<0.001$ & $1.07(0.69-1.65)$ & 0.76 \\
\hline $\mathrm{HBV}+\mathrm{HCV}$ & $0.79(0.25-2.49)$ & 0.69 & & \\
\hline Others $^{\dagger}$ & $1.09(0.73-1.64)$ & 0.67 & & \\
\hline MELD score & $1.16(1.12-1.21)$ & $<0.001$ & $1.10(0.99-1.23)$ & 0.09 \\
\hline AST, IU/L & $1.01(1.004-1.010)$ & $<0.001$ & $1.00(0.997-1.006)$ & 0.58 \\
\hline ALT, IU/L & $1.00(0.998-1.004)$ & 0.49 & & \\
\hline Albumin, g/dL & $0.33(0.26-0.43)$ & $<0.001$ & $0.57(0.42-0.79)$ & $<0.001$ \\
\hline Total bilirubin, mg/dL & $1.12(1.03-1.21)$ & 0.01 & $0.92(0.72-1.17)$ & 0.49 \\
\hline Creatinine, mg/dL & $1.15(1.01-1.33)$ & 0.04 & $0.93(0.64-1.34)$ & 0.68 \\
\hline PT INR & $8.21(4.02-16.76)$ & $<0.001$ & $0.56(0.14-2.25)$ & 0.41 \\
\hline Platelet, $\times 10^{3} / \mu \mathrm{L}$ & 0.995 (0.992-0.997) & $<0.001$ & $1.00(0.997-1.003)$ & 0.90 \\
\hline AFP, log (ng/mL) & $1.13(1.08-1.18)$ & $<0.001$ & $1.04(0.99-1.11)$ & 0.13 \\
\hline Tumor size, cm & $1.13(0.998-1.278)$ & 0.05 & & \\
\hline No. of tumor & $1.12(0.81-1.54)$ & 0.50 & & \\
\hline \multicolumn{5}{|l|}{ Treatment method } \\
\hline Resection vs RFA & $0.80(0.61-1.07)$ & 0.13 & & \\
\hline \multicolumn{5}{|l|}{ Recurrence, yr } \\
\hline \multicolumn{5}{|l|}{$0^{\ddagger}$} \\
\hline$<1$ & $47.42(19.43-115.75)$ & $<0.001$ & 36.91 (15.02-90.69) & $<0.001$ \\
\hline $1-2$ & $18.83(7.45-47.57)$ & $<0.001$ & $15.88(6.26-40.27)$ & $<0.001$ \\
\hline $2-3$ & $16.11(6.07-42.73)$ & $<0.001$ & $13.04(4.88-34.82)$ & $<0.001$ \\
\hline$\geq 3$ & $4.72(1.57-14.17)$ & 0.006 & $4.41(1.46-13.29)$ & 0.008 \\
\hline
\end{tabular}

HR, hazard ratio; CI, confidence interval; HCV, hepatitis C virus; HBV, hepatitis B virus; MELD, Model for End-Stage Liver Disease; AST, aspartate aminotransferase; ALT, alanine aminotransferase; PT INR, international normalized ratio for prothrombin time; AFP, alpha-fetoprotein; RFA, radiofrequency ablation.

*p-value estimated by Cox proportional hazard regression; ${ }^{\dagger}$ Other causes included alcohol, non-alcoholic fatty liver disease, primary biliary cholangitis, and autoimmune hepatitis; ${ }^{\ddagger}$ Reference: non-recurrence. 
a follow-up (living patients) of 1 day. Survival and recurrence were analyzed as the estimated lead time. Survival curves were analyzed via Kaplan-Meier methods and compared with log rank tests. To identify risk factors for overall mortality, univariable and multivariable analyses were performed.

To develop a new model for predict recurrence, study population was randomly divided into the training and validation cohort (2:1 ratio). To select best predictors and derive a new model in the training cohort, backward elimination methods were performed. For selecting and internal validation, bootstrapping procedure was applied with 1,000 bootstrap samples. In external validation, the model developed in the derivation cohort was applied to the validation cohort.

Propensity score matching was performed to reduce selection bias and confounders between 3-monthly and 6-monthly surveillance groups at baseline. To derive propensity scores, the following variables were included in a multiple logistic regression: age, gender, etiologies, CTP score, Model for End-Stage Liver Disease (MELD) score, serum aspartate aminotransferase, alanine aminotransferase, albumin, total bilirubin, creatinine, international normalized ratio for prothrombin time (PT INR), platelet counts, serum AFP levels, tumor size, tumor number, BCLC stage, and treatment methods. One-to-one propensity score matching was performed by nearest neighbor matching with a caliper width of 0.1 multiplied by the standard deviation of the linearly transformed propensity scores. For the propensity score-matched cohort, differences in demographic and clinical characteristics were examined with a paired t-test for continuous variables and McNemar test for categorical variables.

In order to stratify high or low risk on HCC recurrence, the classification and regression tree was conducted. All statistical analyses were performed within the R statistical programming version 3.3.1 (Vienna, Austria, http://www.r-project.org). A pvalue $<0.05$ was considered to indicate a statistically significant difference.

\section{RESULTS}

\section{Baseline characteristics}

During the study period, a total of 1,316 patients were included (Table 1). The median age was 58 years (interquartile range [IQR], 52 to 65 years) and 988 patients (75.1\%) were male. A median CTP score and MELD score were 5 (IQR, 5 to 5 ) and 8 (IQR, 7 to 10), respectively. The median diameter of the largest measurable lesion was $2.3 \mathrm{~cm}$ (IQR, 1.7 to $3.0 \mathrm{~cm}$ ). The number of baseline measurable lesions was one in 1,145 patients (87.0\%). The median AFP level was $11.3 \mathrm{ng} / \mathrm{mL}$ (IQR, 5.0 to $94.3 \mathrm{ng} / \mathrm{mL}$ ), 859 patients (65.3\%) had the BCLC stage A and 812 patients (61.7\%) and 504 patients (38.3\%) underwent RFA and resection, respectively. During the study period (median, 40 months; IQR, 20 to 64 months), survival rates were $76.5 \%$ at 5 years and a total of 766 patients (58.1\%) had recurred HCC in the total population. Complication of cirrhosis such as ascites, variceal bleeding, or hepatic encephalopathy was developed in 165 patients (12.5\%).

Among the total population, patients who did not recur within 1 year after treatment were randomly divided to two groups: the training cohort $(66.7 \%, n=582)$ and the validation cohort (33.3\%, $n=291)$. In the training cohort, the median age was 58 years (IQR, 52 to 65 years) and 74.1\% were male. A median CTP score and MELD score were 5 (IQR, 5 to 5) and 8 (IQR, 7 to 9), respectively. The median diameter of the largest measurable lesion was $2.3 \mathrm{~cm}$ (IQR, 1.6 to $3.0 \mathrm{~cm}$ ). The number of baseline measurable lesions was one in $88.8 \%$ of the patients. The median AFP level was $10.2 \mathrm{ng} / \mathrm{mL}$ (IQR, 4.9 to $76.0 \mathrm{ng} / \mathrm{mL}$ ). $62.7 \%$ of the patients had the BCLC stage A. $61.2 \%$ and $38.8 \%$ of patients underwent RFA and resection, respectively. The validation cohort had similar baseline characteristics compared to the training cohort except MELD scores (all except MELD scores p>0.05) (Table 1).

\section{Risk factors for overall mortality}

Multivariable analysis to identify risk factors related to overall mortality was performed among the patients under 3-monthly surveillance ( $\mathrm{n}=915)$. Serum albumin levels and recurrence status were significant independent factors for overall mortality (Table 2). The modalities of curative treatment were not significant independent predictors for overall mortality (hazard ratio [HR], 0.80; 95\% confidence interval [CI], 0.61 to $1.07 ; \mathrm{p}=0.13$ as resection compared with RFA).

Regarding recurrence-free durations, the HR of each recurrence-free duration for overall mortality was different: 36.91 (95\% CI, 15.02 to $90.69 ; \mathrm{p}<0.001$ ) at recurrence $<1$ year, 15.88 ( $95 \%$ CI, 6.26 to $40.27 ; \mathrm{p}<0.001$ ) at recurrence of 1 to 2 years, 13.04 ( $95 \%$ CI, 4.88 to $34.82 ; \mathrm{p}<0.001$ ) at recurrence of 2 to 3 years, and 4.41 (95\% CI, 1.46 to $13.29 ; \mathrm{p}=0.008)$ at recurrence $>3$ years compared to non-recurrence (Table 2). Recurrence-free durations $<1$ year showed a significantly higher HR for overall mortality than other recurrence-free durations such as 1 to 2 years, 2 to 3 years, and $>3$ years (all, $p<0.001$ ). Recurrence-free durations of 2 to 3 years were not significantly lower risk for OS

Table 3. Comparison of Risk of Each Recurrence-Free Duration for Survival Rates $(\mathrm{n}=1,295)$

\begin{tabular}{ccc}
\hline Recurrence-free duration & HR $(95 \% \mathrm{CI})$ & p-value $^{*}$ \\
\hline$<1$ yr vs 1-2 yr & $0.44(0.31-0.63)$ & $<0.001$ \\
$<1$ yr vs 2-3 yr & $0.35(0.22-0.55)$ & $<0.001$ \\
$<1$ yr vs $>3$ yr & $0.12(0.06-0.23)$ & $<0.001$ \\
$1-2$ yr vs 2-3 yr & $0.79(0.46-1.33)$ & 0.37 \\
$1-2$ yr vs $>3$ yr & $0.27(0.13-0.55)$ & $<0.001$ \\
$2-3$ yr vs $>3$ yr & $0.34(0.15-0.75)$ & 0.007 \\
\hline
\end{tabular}

$\mathrm{HR}$, hazard ratio; CI, confidence interval.

*p-value estimated by Cox proportional hazard regression method. 
than recurrence-free durations of 1 to 2 years ( $\mathrm{HR}, 0.79 ; 95 \%$ CI, 0.46 to $1.33 ; \mathrm{p}=0.37$ ) (Table 3 ).

\section{Development and validation of the risk prediction model}

Two significant predictors from back elimination analyses such as age and PT INR levels were selected to generate the risk prediction model for recurrence (Table 4). Assessment for Surveillance interval score (AS score) was defined as the sum of the weighted variables: AS score $=0.176 \times$ age (year)+17.279 $\times$ PT INR-21.887. The model showed 0.62 of area under the receiver operating characteristic curve (AUROC) for recurrence at 5 year (95\% CI, 0.56 to 0.69) in the training cohort and 0.64 of AUROC for recurrence at 5 year (95\% CI, 0.55 to 0.73 ) in the validation cohort. According to the AS scores, patients were stratified into two groups; for example, low-risk groups (AS score <9.26) and high-risk groups (AS score $\geq 9.26$ ). The high-risk group showed significantly higher recurrence rates than the low-risk group in the training cohort (HR, 1.55; 95\% CI, 1.17 to 2.07, $\mathrm{p}=0.003$ ) and the validation cohort (HR, 1.73; 95\% CI, 1.18 to 2.53 ; $\mathrm{p}=0.005$ ) (Fig. 2A and B).

Regarding prediction of overall mortality in total population, the model showed 0.70 of AUROC at 5 year (95\% CI, 0.63 to $0.76)$ and significantly stratified the individual risk of overall mortality: the low-risk group showed significantly higher survival rates than the high-risk group (HR, 3.84; 95\% CI, 2.37 to 6.22; $\mathrm{p}<0.001$ ) (Fig. 2C). Regarding prediction of complications of liver cirrhosis in total population, the model showed 0.74 of AUROC at 5 year (95\% CI, 0.67 to 0.80 ) and significantly stratified the individual risk of cirrhotic complications among HCC patients with BCLC 0/A stages: the high-risk group showed significantly higher cirrhotic complication rates than the low-risk group (HR, 4.12; 95\% CI, 2.49 to 6.81; p<0.001) (Fig. 2D).

\section{Comparison of survival rates in propensity-score matched cohorts between 3-monthly and 6-monthly surveillance from the first year after treatment}

Among patients who all underwent CT scans every 3 months until recurrence, low-risk patients $(n=376)$ were identified based on AS scores (AS score <9.26); among patients followed-up every 3 months for 1 year after curative treatment and every 6 months thereafter, low-risk patients $(n=314)$ were identified. After propensity-score matching between the two groups, 140

Table 4. Selection of Variables for a New Model Using Backward Elimination Method in the Training Cohorts ( $\mathrm{n}=582)$

\begin{tabular}{|c|c|c|c|c|}
\hline \multirow{2}{*}{ Variable } & \multicolumn{2}{|c|}{ Univariable } & \multicolumn{2}{|c|}{ Multivariable } \\
\hline & HR (95\% CI) & p-value* & HR $(95 \% \mathrm{CI})$ & p-value* \\
\hline Age, yr & $1.02(1.003-1.030)$ & 0.02 & $1.028(1.004-1.031)$ & 0.01 \\
\hline Male sex & $1.48(1.09-2.01)$ & 0.01 & - & - \\
\hline Etiology & & & - & - \\
\hline HBV & Reference & & & \\
\hline $\mathrm{HCV}$ & $1.40(0.92-2.13)$ & 0.11 & & \\
\hline $\mathrm{HBV}+\mathrm{HCV}$ & $1.54(0.72-3.27)$ & 0.26 & & \\
\hline Others $^{\dagger}$ & $0.83(0.57-1.21)$ & 0.33 & & \\
\hline MELD score & $1.09(1.04-1.15)$ & $<0.001$ & - & - \\
\hline AST, U/L & $1.01(1.003-1.011)$ & $<0.001$ & - & - \\
\hline ALT, U/L & $1.00(1.001-1.006)$ & 0.01 & - & - \\
\hline Albumin, $\mathrm{g} / \mathrm{dL}$ & $0.59(0.43-0.81)$ & 0.001 & - & - \\
\hline Total bilirubin, mg/dL & $1.14(0.87-1.49)$ & 0.35 & - & - \\
\hline Creatinine, $\mathrm{mg} / \mathrm{dL}$ & $1.09(0.97-1.23)$ & 0.14 & - & - \\
\hline PT INR & $5.20(2.06-13.14)$ & $<0.001$ & $5.63(2.24-14.18)$ & $<0.001$ \\
\hline Platelet, $\times 10^{3} / \mu \mathrm{L}$ & 0.995 (0.992-0.998) & $<0.001$ & - & - \\
\hline AFP, log $(\mathrm{ng} / \mathrm{mL})$ & $1.00(1.00-1.00)$ & 0.73 & - & - \\
\hline Tumor size, cm & $0.95(0.85-1.07)$ & 0.43 & - & - \\
\hline No. of tumor & $1.10(0.39-3.09)$ & 0.85 & - & - \\
\hline \multicolumn{5}{|l|}{ Treatment method } \\
\hline Resection vs RFA & $0.64(0.49-0.83)$ & $<0.001$ & - & - \\
\hline
\end{tabular}

HR, hazard ratio; CI, confidence interval; HBV, hepatitis B virus; HCV, hepatitis C virus; MELD, Model for End-Stage Liver Disease; AST, aspartate aminotransferase; ALT, alanine aminotransferase; PT INR, international normalized ratio for prothrombin time; AFP, alpha-fetoprotein; RFA, radiofrequency ablation.

*p-value estimated by Cox proportional hazard regression; ${ }^{\dagger}$ Other causes included alcohol, non-alcoholic fatty liver disease, primary biliary cholangitis, and autoimmune hepatitis. 

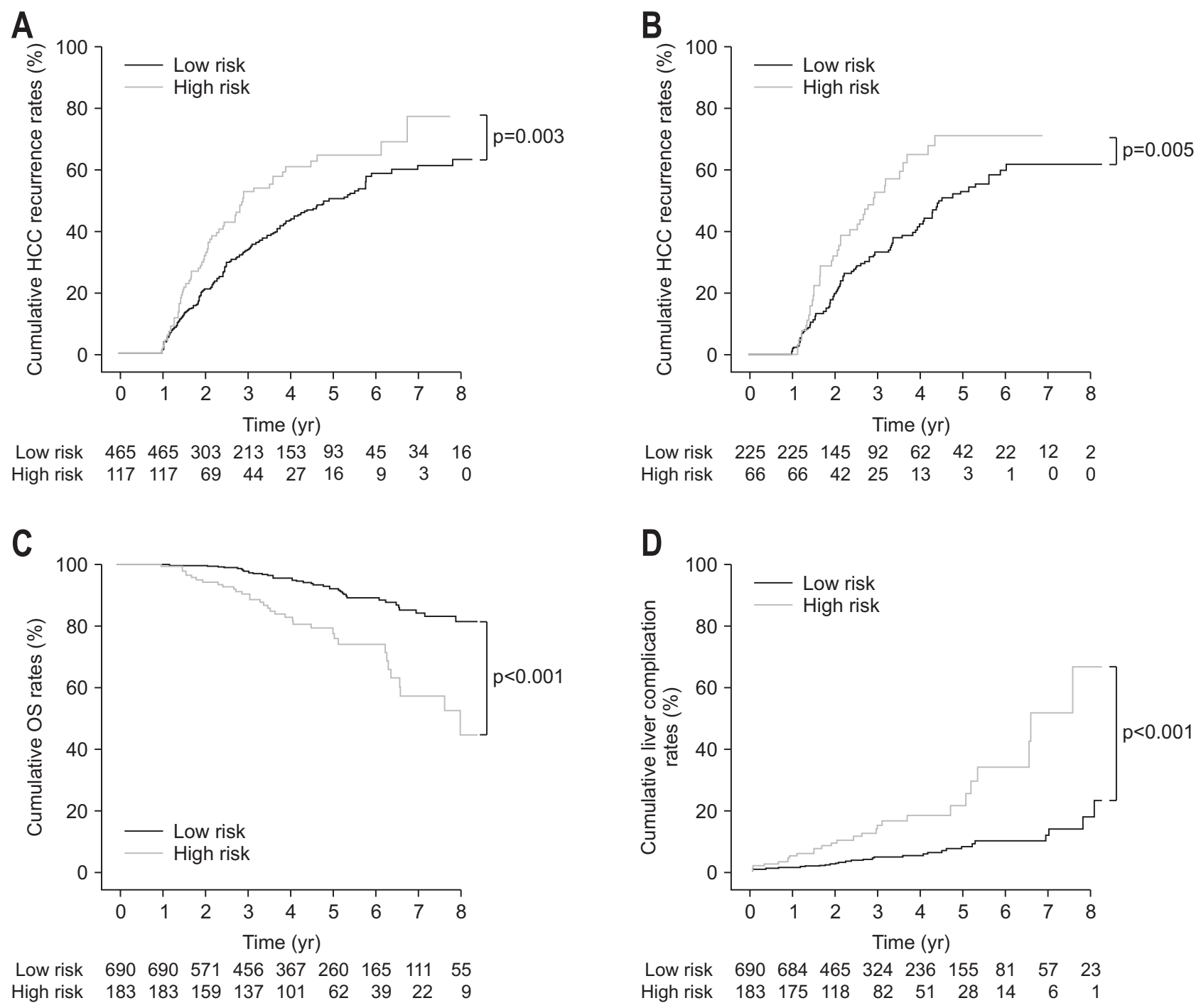

Fig. 2. Clinical outcomes of low- and high-risk patients based on assessment for surveillance interval scores. (A) In the training cohort, the cumulative recurrence rates of hepatocellular carcinoma (HCC) in high-risk patients were significantly higher than those in low-risk patients ( $\mathrm{p}=0.003)$. (B) In the validation cohort, the cumulative recurrence rates of HCC in high-risk patients were significantly higher than those in low-risk patients $(\mathrm{p}=0.005)$. (C) In the total population, overall survival (OS) rates in low-risk patients were significantly higher than those in high-risk patients $(\mathrm{p}<0.001)$. (D) In the total population, the complication rates of cirrhosis in high-risk patients were significantly higher than those in low-risk patients $(\mathrm{p}<0.001)$.

pairs were generated. In the low-risk groups after propensityscore matching ( $\mathrm{n}=280,140$ pairs), there was no significant difference in baseline characteristics between 3-monthly and 6-monthly surveillance groups (Table 5). In propensity-score matching of 3-monthly and 6-monthly surveillance groups, in low-risk patients (AS score <9.26), OS under 6-monthly surveillance were not significantly different from those under 3-monthly surveillance ( $\mathrm{p}=0.17$ by log-rank test) (Fig. 3A).

Among patients who all underwent CT scans every 3 months until recurrence, high-risk patients $(n=96)$ were identified based on AS scores (AS score $\geq 9.26$ ) ; among patients followed up every 3 months for year 1 after curative treatment and every 6 months thereafter, high-risk patients $(n=87)$ were identified. After propensity-score matching between the two groups, 62 pairs were generated. In the high-risk groups after propensityscore matching ( $n=124,62$ pairs), there was no significant difference in baseline characteristics between 3-monthly and 6-monthly surveillance groups except serum ALT levels (Table 6). In propensity-score matching of 3-monthly and 6-monthly surveillance groups, survival rates in high-risk patients (AS score 29.26) under 6-monthly surveillance were significantly lower than those under 3-monthly surveillance ( $\mathrm{p}=0.04$ by log-rank test) (Fig. 3B).

\section{DISCUSSION}

In this study, we developed and validated a risk prediction model based on universally recognized clinical variables not 
Table 5. Baseline Characteristics of Low-Risk Group Under 3-Monthly and 6-Monthly Surveillance from Year 1 after Treatment

\begin{tabular}{|c|c|c|c|c|c|c|}
\hline \multirow{2}{*}{ Variable } & \multicolumn{3}{|c|}{ Original set } & \multicolumn{3}{|c|}{ Matched set } \\
\hline & $\begin{array}{l}\text { 3-Monthly surveillance } \\
\qquad(\mathrm{n}=376)\end{array}$ & $\begin{array}{l}\text { 6-Monthly surveillance } \\
\qquad(\mathrm{n}=314)\end{array}$ & p-value ${ }^{*}$ & $\begin{array}{l}\text { 3-Monthly surveillance } \\
\qquad(\mathrm{n}=140)\end{array}$ & $\begin{array}{l}\text { 6-Monthly surveillance } \\
\qquad(\mathrm{n}=140)\end{array}$ & p-value ${ }^{*}$ \\
\hline Age, yr & $57(51-62)$ & $56(50-63)$ & 0.34 & $56(50-62)$ & $56(51-63)$ & 0.72 \\
\hline Male sex & $306(81.4)$ & $219(69.8)$ & $<0.001$ & $100(71.4)$ & 95 (67.9) & 0.60 \\
\hline Etiology & & & 0.59 & & & 0.21 \\
\hline HBV & $309(82.2)$ & 251 (79.9) & & $120(85.7)$ & $108(77.2)$ & \\
\hline HCV & $17(4.5)$ & $11(3.5)$ & & $7(5.0)$ & $7(5.0)$ & \\
\hline $\mathrm{HBV}+\mathrm{HCV}$ & $7(1.9)$ & $6(1.9)$ & & $2(1.4)$ & $3(2.1)$ & \\
\hline Others ${ }^{\dagger}$ & $43(11.4)$ & $46(14.7)$ & & $11(7.9)$ & $22(15.7)$ & \\
\hline CTP score & $5(5-5)$ & $5(5-5)$ & 0.71 & $5(5-5)$ & $5(5-5)$ & 0.81 \\
\hline MELD score & $8(7-9)$ & $8(7-9)$ & 0.39 & $8(7-9)$ & $8(7-8)$ & 0.19 \\
\hline AST, U/L & $34(26-43)$ & $32(25-44)$ & 0.26 & $35(27-44)$ & $31(36-43)$ & 0.19 \\
\hline ALT, U/L & $35(24-51)$ & 33 (22-49) & 0.28 & $35(25-53)$ & $32(23-51)$ & 0.27 \\
\hline Albumin, g/dL & $4.1(3.9-4.4)$ & $4.1(3.9-4.4)$ & 0.66 & $4.2(3.9-4.4)$ & $4.1(3.9-4.3)$ & 0.22 \\
\hline Total bilirubin, mg/dL & $0.9(0.7-1.1)$ & $0.9(0.7-1.1)$ & 0.51 & $0.9(0.8-1.1)$ & $0.9(0.7-1.1)$ & 0.14 \\
\hline Creatinine, mg/dL & $0.9(0.8-1.1)$ & $0.9(0.8-1.1)$ & 0.82 & $1.0(0.8-1.1)$ & $0.9(0.8-1.1)$ & 0.29 \\
\hline PT INR & $1.1(1.0-1.1)$ & $1.1(1.0-1.1)$ & 0.40 & $1.1(1.0-1.1)$ & $1.1(1.0-1.1)$ & 0.40 \\
\hline Platelet, $\times 10^{3} / \mu \mathrm{L}$ & $142(108-177)$ & $148(108-183)$ & 0.41 & $132(109-172)$ & $151(110-184)$ & 0.08 \\
\hline AFP, ng/mL & $8.9(4.2-52.4)$ & $10.0(4.5-117.0)$ & 0.30 & $8.9(4.2-80.6)$ & $10.8(4.8-102.0)$ & 0.44 \\
\hline Tumor size, cm & $2.3(1.7-3.0)$ & $2.3(1.6-3.4)$ & 0.18 & $2.3(1.6-3.0)$ & $2.2(1.7-3.0)$ & 0.51 \\
\hline No. of tumor & & & 0.35 & & & 1.00 \\
\hline 1 & $330(87.8)$ & $286(91.1)$ & & $125(89.3)$ & $126(90.0)$ & \\
\hline 2 & $38(10.1)$ & $24(7.6)$ & & $13(9.3)$ & $12(8.6)$ & \\
\hline 3 & $8(2.1)$ & $4(1.3)$ & & $2(1.4)$ & $2(1.4)$ & \\
\hline BCLC stage & & & 0.92 & & & 0.39 \\
\hline 0 & $140(37.2)$ & 119 (37.9) & & $52(37.1)$ & 60 (42.9) & \\
\hline $\mathrm{A}$ & $236(62.8)$ & $195(62.1)$ & & $88(62.9)$ & $80(57.1)$ & \\
\hline Treatment method & & & 0.02 & & & 0.55 \\
\hline RFA & $226(60.1)$ & $160(51.0)$ & & $84(60.0)$ & 78 (55.7) & \\
\hline Resection & $150(39.9)$ & $154(49.0)$ & & $56(40.0)$ & $62(44.3)$ & \\
\hline
\end{tabular}

Data are presented as median (interquartile range) or number (\%).

HBV, hepatitis B virus; HCV, hepatitis C virus; CTP, Child-Turcotte-Pugh; MELD, Model for End-Stage Liver Disease; AST, aspartate aminotransferase; ALT, alanine aminotransferase; PT INR, international normalized ratio for prothrombin time; AFP, alpha-fetoprotein; BCLC, Barcelona Clinic Liver Cancer; RFA, radiofrequency ablation.

*p-value estimated by chi-square test or Fisher exact test for categorical variables and Mann-Whitney U test; ${ }^{\dagger}$ Other causes included alcohol, nonalcoholic fatty liver disease, primary biliary cholangitis, and autoimmune hepatitis.

only for improved accuracy in predicting prognostic outcomes in patients with very early or early HCC undergoing treatments with curative intent but also for guidance on extending the surveillance interval in some patients. Our results support reducing the frequency of surveillance CT studies in low-risk patients without incurring significant hazard on survival rates, compared to those under continuous 3-month interval surveillance, and we suggest 3-monthly surveillance in high-risk patients.

We found that both the aggressive tumor biology such as early recurrence, and relatively poor liver function such as lower serum albumin levels were associated with a dismal prognosis for patients who were curatively treated with surgery or RFA even in the BCLC 0/A group. In our Cox regression analysis, recurrence-free duration ( $\leq 1$ year) showed the highest HR for overall mortality among the significant variables. Particularly, time point of year 1 after treatment was a more important time point to extend the surveillance intervals for survival than time point of year 2: 1-year recurrence showed significantly higher HR for overall mortality compared to other recurrencefree durations (vs $1-2,2-3, \geq 3$ years). Therefore, we designed a new model to guide the surveillance interval at year 1 after treatments, rather than other time points, such as $\geq 2$ years after 

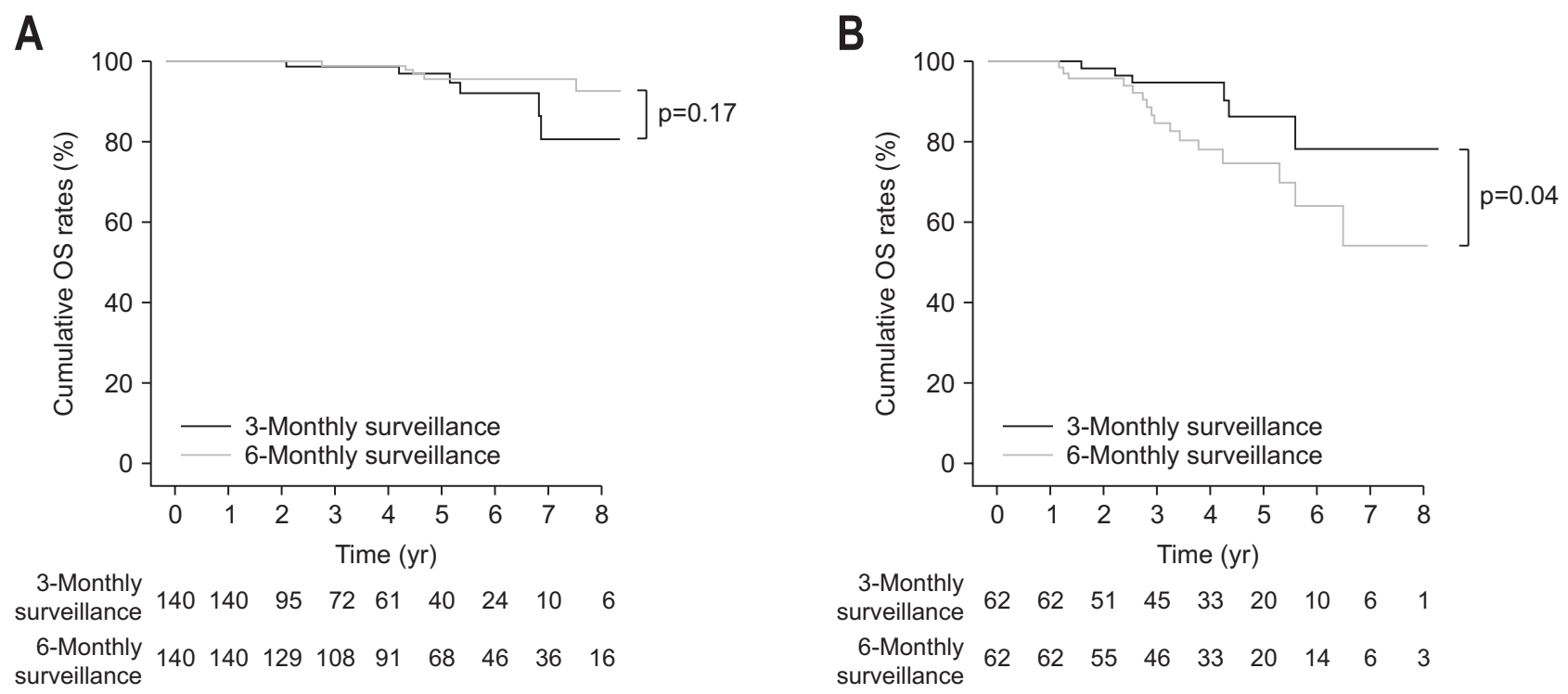

Fig. 3. Comparison of survival rates after propensity-score matching between patients under 6-monthly surveillance 1 year after curative treatment and those under 3-monthly surveillance. (A) The cumulative survival rates of low-risk patients under 6-monthly surveillance 1 year after curative treatment were not significantly different from those under 3-monthly surveillance ( $\mathrm{p}=0.17)$. (B) The cumulative survival rates of highrisk patients under 3-monthly surveillance were significantly higher than those of high-risk patients under 6-monthly surveillance 1 year after curative treatment $(\mathrm{p}=0.04)$.

treatment in patients with a BCLC 0/A stage. In addition, when recurrence periods were also divided by the cutoff period of 2 years, late recurrence ( $>2$ years) showed significantly lower overall mortality than did early recurrence ( $\leq 2$ years) (HR, 0.19; 95\% CI, 0.13 to 0.28 ; $p<0.001)$. Although the cutoff duration of 2 years also showed significant stratification of prognosis for survival, our subgroup analysis in this study revealed that recurrence within 1 year showed significantly lower survival than did that in periods between 1 year and 2 years (Table 3), and the recurrence pattern was changed after 1 year (Supplementary Fig. 1)

Among patients who did not recur within year 1, significant variables to predict recurrence were older age and higher PT INR levels. These factors did not reflect tumor biology such as tumor size, tumor number, and AFP levels. This can be explained by recurrence within year 1 after treatment itself showed the highest HR for overall mortality among any other risk factors such tumor size, number, and AFP levels.

Treatment modalities such as resection and RFA were not significant factors for survival in the multivariable analyses for overall mortality and not selected as significant variables. Because enrolled patients in this study had small-sized tumors and very early or early stages of HCC, we think that the modalities of curative treatment could not be significant independent factors for overall mortality. Tumor stages in this study were limited to BCLC stage 0/A. The median tumor size was $2.3 \mathrm{~cm}$, and the tumor number in most of patients (87\%) was only one. In patients with a single, small-sized tumor, many previous studies, including randomized controlled trials and meta-analyses, indicated that survival rates in BCLC 0/A patients treated with surgery were not significantly different from those in patients treated with RFA. ${ }^{18-21}$ We think that our results were consistent with previous studies.

In this study, serum AFP levels were relatively low compared with those in previous studies. An explanation for this result is that the target population in this study included the patients with very early or early stages of HCC. The reason that the AS score did not include tumor factors, such as tumor size, number, and serum AFP levels, was that the AS score was derived from patients without 1-year recurrence after treatment, who, in other words, had a relatively good prognosis. Given that the aim of our study was to guide the surveillance strategies for patients without 1-year recurrence at 1 year after treatment, our findings can explain why the AS score consisted of only host factors, not tumor factors.

Based on the risk stratification, AS scores can be used to identify patients who may have extended surveillance intervals from 3 months to 6 months at year 1 after curative treatment. Among patients who maintain 3-monthly surveillance, low-risk patients with $<9.26$ points as an AS score had a good prognosis. This subgroup did not show any significant difference in OS for patients who extended surveillance intervals to 6 months at year 1 after treatments. In contrast, when patients were identified as the high-risk group with an AS score $\geq 9.26$, patients under 6-monthly surveillance showed lower OS than those under 3-monthly surveillance. Thus, at year 1 after curative treatment, high-risk patients should maintain 3-monthly surveillance rather than changing to extend the interval. 
Table 6. Baseline Characteristics of High-Risk Group under 3-Monthly and 6-Monthly Surveillance from Year 1 after Treatment

\begin{tabular}{|c|c|c|c|c|c|c|}
\hline \multirow{2}{*}{ Variable } & \multicolumn{3}{|c|}{ Original set } & \multicolumn{3}{|c|}{ Matched set } \\
\hline & $\begin{array}{l}\text { 3-Monthly surveillance } \\
\qquad(\mathrm{n}=96)\end{array}$ & $\begin{array}{l}\text { 6-Monthly surveillance } \\
\text { (n=87) }\end{array}$ & p-value ${ }^{*}$ & $\begin{array}{l}\text { 3-Monthly surveillance } \\
\qquad(\mathrm{n}=62)\end{array}$ & $\begin{array}{l}\text { 6-Monthly surveillance } \\
\qquad(\mathrm{n}=62)\end{array}$ & p-value ${ }^{*}$ \\
\hline Age, yr & $67(61-73)$ & 66 (61-70) & 0.20 & $66(59-71)$ & $66(61-72)$ & 0.62 \\
\hline Male sex & 69 (71.9) & 51 (58.6) & 0.08 & $44(71.0)$ & 37 (59.7) & 0.26 \\
\hline Etiology & & & 0.81 & & & 0.89 \\
\hline HBV & $47(49.0)$ & $47(54.0)$ & & $33(53.2)$ & $32(51.6)$ & \\
\hline $\mathrm{HCV}$ & $26(27.1)$ & 24 (27.6) & & $15(24.2)$ & $17(27.4)$ & \\
\hline $\mathrm{HBV}+\mathrm{HCV}$ & $1(1.0)$ & $1(1.2)$ & & $0(0.0)$ & $1(1.6)$ & \\
\hline Others $^{\dagger}$ & $22(22.9)$ & $15(17.2)$ & & $14(22.6)$ & $12(19.4)$ & \\
\hline CTP score & $5(5-6)$ & $5(5-6)$ & 0.56 & $5(5-6)$ & $5(5-6)$ & 0.82 \\
\hline MELD score & $10(9-11)$ & $10(9-11)$ & 0.28 & $10(9-11)$ & $10(9-11)$ & 0.76 \\
\hline AST, U/L & $47(33-64)$ & 47 (34-57) & 0.53 & $47(34-56)$ & $47(35-55)$ & 0.65 \\
\hline ALT, U/L & 40 (29-65) & 34 (24-48) & 0.008 & $42(30-57)$ & 34 (24-49) & 0.03 \\
\hline Albumin, g/dL & $3.9(3.50-4.1)$ & $3.8(3.5-4.0)$ & 0.32 & $3.8(3.5-4.1)$ & $3.8(3.5-4.1)$ & 0.94 \\
\hline Total bilirubin, mg/dL & $1.1(0.9-1.4)$ & $1.2(1.0-1.5)$ & 0.49 & $1.2(0.9-1.5)$ & $1.2(1.0-1.4)$ & 0.97 \\
\hline Creatinine, mg/dL & $0.9(0.8-1.0)$ & $0.9(0.8-1.0)$ & 0.74 & $0.9(0.8-1.0)$ & $0.9(0.8-1.0)$ & 0.81 \\
\hline PT INR & $1.2(1.1-1.3)$ & $1.2(1.2-1.3)$ & 0.26 & $1.2(1.2-1.3)$ & $1.2(1.2-1.3)$ & 0.88 \\
\hline Platelet, $\times 10^{3} / \mu \mathrm{L}$ & $109(74-160)$ & $91(65-123)$ & 0.02 & $93(71-121)$ & $93(68-126)$ & 0.76 \\
\hline AFP, ng/mL & $11.4(5.5-55.4)$ & $13.0(6.0-32.5)$ & 0.82 & $9.0(5.1-21.0)$ & $13.9(5.9-34.0)$ & 0.25 \\
\hline Tumor size, cm & $2.1(1.6-2.8)$ & $2.1(1.8-2.9)$ & 0.78 & $2.0(1.5-2.8)$ & $2.1(1.7-2.9)$ & 0.61 \\
\hline Tumor number & & & 0.70 & & & 1.00 \\
\hline 1 & 86 (89.6) & $74(85.1)$ & & $53(85.5)$ & $54(87.1)$ & \\
\hline 2 & $8(8.3)$ & $11(12.6)$ & & $7(11.3)$ & $6(9.7)$ & \\
\hline 3 & $2(2.1)$ & $2(2.3)$ & & $2(3.2)$ & $2(3.2)$ & \\
\hline BCLC stage & & & 1.00 & & & 1.00 \\
\hline 0 & $37(38.5)$ & $34(39.1)$ & & $25(40.3)$ & $26(41.9)$ & \\
\hline A & $59(61.5)$ & $53(60.9)$ & & 37 (59.7) & $36(58.1)$ & \\
\hline Treatment method & & & 0.88 & & & 1.00 \\
\hline RFA & $72(75.0)$ & $67(77.0)$ & & $47(75.8)$ & $48(77.4)$ & \\
\hline Resection & $24(25.0)$ & $20(23.0)$ & & $15(24.2)$ & $14(22.6)$ & \\
\hline
\end{tabular}

Data are presented as median (interquartile range) or number (\%).

HBV, hepatitis B virus; HCV, hepatitis C virus; CTP, Child-Turcotte-Pugh; MELD, Model for End-Stage Liver Disease; AST, aspartate aminotransferase; ALT, alanine aminotransferase; PT INR, international normalized ratio for prothrombin time; AFP, alpha-fetoprotein; BCLC, Barcelona Clinic Liver Cancer; RFA, radiofrequency ablation.

*p-value estimated by chi-square test or Fisher exact test for categorical variables and Mann-Whitney U test; ${ }^{\dagger}$ Other causes included alcohol, nonalcoholic fatty liver disease, primary biliary cholangitis, and autoimmune hepatitis.

Although it is difficult to conclude how long 3-monthly surveillance in the high-risk patients will be maintained in this study because of small patient numbers, it can be assumed that 3-monthly surveillance in the high-risk patients might be helpful up to year 3 after curative treatment: patients without 3-year recurrence (37.2\%) after curative treatments in the high-risk group showed 5-year survival rates of 93.5\% in patients under 3-monthly surveillance, which were not significantly different from 5-year survival rates of 90.0\% in patients under 6-monthly surveillance at year 1 after treatments. However, high-risk patients under 6-monthly surveillance without 1- or 2-year re- currence showed significantly lower survival rates compared to those under 3-monthly surveillance.

These findings have clinical relevance for several reasons. First, the AS score is easily applicable in real-life clinical settings even in countries with limited healthcare resources. Second, the application of AS scores may provide a rationale for extended intervals in low-risk patients and guide physicians to optimize surveillance intervals at year 1 after curative treatment. Third, the use of AS scores may prevent radio-hazards and costs by over-surveillance using frequent CT scans without compromising the survival benefits in low-risk patients. In con- 
trast, $21.0 \%$ of patients who received curative treatment were stratified in the high-risk group by AS scores and those patients need to cautiously maintain stringent surveillance due to low survival rates compared to those in the low-risk group.

A major limitation of the current study is that it was based on retrospective observational data. However, in the case of surveillance for HCC, prospective, randomized, controlled studies are almost impossible to perform in regions where easy access to diagnostic tools raises ethical problems and makes patient compliance to the physician very unlikely. Second, AS scores did not reflect pathological factors, which have been reported as significant predictors for recurrence, such as microvascular invasion or satellite nodules in patients treated with resection. ${ }^{22}$ Although microvascular invasion was one of the important independent factors for recurrence, inter/intra-observer variability in reporting, the lack of definition, and grading of microvascular invasion have led to great heterogeneity in evaluating this histological feature in HCC. ${ }^{23,24}$ Furthermore, it is impossible to get pathology data in most patients treated with RFA in real clinical setting. Given that AS scores are derived from patients who received curative treatments such as RFA or surgery and are composed of all objective factors, it might be more practical and objective in real fields in terms of error possibilities from the subjective aspects of equivocal pathological findings. ${ }^{23,24}$ Third, unfortunately, we could not obtain information about details of liver-related mortality from complications of liver cirrhosis in this cohort because overall mortality data, not causespecific mortality data, could be obtained from the Korean National Health Insurance Service. It needs further validation study regarding the prediction of liver-related mortality using the proposed model in the future. However, generally, in previous reports studying surveillance benefits in HCC patients, primary endpoint was overall survival. ${ }^{25-27}$ Based on their results, overall survival as primary endpoint in our study can be applicable to conclude the surveillance benefit in HCC patients. Lastly, there may be selection bias contributed by the subjective choice of the interval. Doctors tend to shorten the interval in patients considered at very high risk of HCC, such as those with larger tumor size, higher MELD scores, and elevated baseline serum AFP levels. ${ }^{28}$ However, in this study, propensity-score matched analyses were performed to minimize selection bias: baseline characteristics between 3-monthly and 6-months surveillance groups were well-balanced.

In conclusion, the AS score developed here was able to stratify risk for HCC recurrence among patients curatively treated, and could therefore provide an objective evidence-based tool to determine the patients who can have an extended surveillance CT interval or maintain stringent surveillance. Future larger prospective validation studies are needed to confirm CT surveillance intervals that are optimized according to the patients' risk of HCC.

\section{CONFLICTS OF INTEREST}

No potential conflict of interest relevant to this article was reported.

\section{ACKNOWLEDGEMENTS}

This research was supported by a grant of the Korea Health Technology R\&D Project through the Korea Health Industry Development Institute (KHIDI), funded by the Ministry of Health \& Welfare, Republic of Korea (grant number: HI16C1074).

\section{REFERENCES}

1. Ikai I, Arii S, Okazaki M, et al. Report of the 17th Nationwide Follow-up Survey of Primary Liver Cancer in Japan. Hepatol Res 2007;37:676-691

2. European Association for the Study of the Liver; European Organisation for Research and Treatment of Cancer. EASL-EORTC clinical practice guidelines: management of hepatocellular carcinoma. J Hepatol 2012;56:908-943.

3. Yu SJ. A concise review of updated guidelines regarding the management of hepatocellular carcinoma around the world: 20102016. Clin Mol Hepatol 2016;22:7-17.

4. Kumada T, Nakano S, Takeda I, et al. Patterns of recurrence after initial treatment in patients with small hepatocellular carcinoma. Hepatology 1997;25:87-92.

5. Adachi E, Maeda T, Matsumata T, et al. Risk factors for intrahepatic recurrence in human small hepatocellular carcinoma. Gastroenterology 1995;108:768-775.

6. Okada S, Shimada K, Yamamoto J, et al. Predictive factors for postoperative recurrence of hepatocellular carcinoma. Gastroenterology 1994;106:1618-1624.

7. Belghiti J, Panis Y, Farges O, Benhamou JP, Fekete F. Intrahepatic recurrence after resection of hepatocellular carcinoma complicating cirrhosis. Ann Surg 1991;214:114-117.

8. Llovet JM, Schwartz M, Mazzaferro V. Resection and liver transplantation for hepatocellular carcinoma. Semin Liver Dis 2005;25:181-200.

9. Balsells J, Charco R, Lazaro JL, et al. Resection of hepatocellular carcinoma in patients with cirrhosis. Br J Surg 1996;83:758-761.

10. Chen MF, Hwang TL, Jeng LB, Wang CS, Jan YY, Chen SC. Postoperative recurrence of hepatocellular carcinoma: two hundred five consecutive patients who underwent hepatic resection in 15 years. Arch Surg 1994;129:738-742.

11. Lau H, Fan ST, Ng IO, Wong J. Long term prognosis after hepatectomy for hepatocellular carcinoma: a survival analysis of 204 consecutive patients. Cancer 1998;83:2302-2311.

12. Takenaka K, Kawahara N, Yamamoto K, et al. Results of 280 liver resections for hepatocellular carcinoma. Arch Surg 1996;131:7176.

13. Bruix J, Sherman M; American Association for the Study of Liver 
Diseases. Management of hepatocellular carcinoma: an update. Hepatology 2011;53:1020-1022.

14. Kim SU, Lee JH, Kim DY, et al. Prediction of liver-related events using fibroscan in chronic hepatitis B patients showing advanced liver fibrosis. PLoS One 2012;7:e36676.

15. Adams PC, Arthur MJ, Boyer TD, et al. Screening in liver disease: report of an AASLD clinical workshop. Hepatology 2004;39:12041212.

16. Schwartz M. A biomathematical approach to clinical tumor growth. Cancer 1961;14:1272-1294.

17. Sheu JC, Sung JL, Chen DS, et al. Growth rate of asymptomatic hepatocellular carcinoma and its clinical implications. Gastroenterology 1985;89:259-266.

18. Ng KK, Chok KS, Chan AC, et al. Randomized clinical trial of hepatic resection versus radiofrequency ablation for early-stage hepatocellular carcinoma. Br J Surg 2017;104:1775-1784.

19. Majumdar A, Roccarina D, Thorburn D, Davidson BR, Tsochatzis E, Gurusamy KS. Management of people with early- or very earlystage hepatocellular carcinoma: an attempted network metaanalysis. Cochrane Database Syst Rev 2017;3:CD011650.

20. Wang Y, Luo Q, Li Y, Deng S, Wei S, Li X. Radiofrequency ablation versus hepatic resection for small hepatocellular carcinomas: a meta-analysis of randomized and nonrandomized controlled trials. PLoS One 2014;9:e84484.

21. Livraghi T, Meloni F, Di Stasi M, et al. Sustained complete response and complications rates after radiofrequency ablation of very early hepatocellular carcinoma in cirrhosis: Is resection still the treatment of choice? Hepatology 2008;47:82-89.

22. Shim JH, Jun MJ, Han S, et al. Prognostic nomograms for prediction of recurrence and survival after curative liver resection for hepatocellular carcinoma. Ann Surg 2015;261:939-946.

23. Rodríguez-Perálvarez M, Luong TV, Andreana L, Meyer T, Dhillon AP, Burroughs AK. A systematic review of microvascular invasion in hepatocellular carcinoma: diagnostic and prognostic variability. Ann Surg Oncol 2013;20:325-339.

24. Fan L, Mac MT, Frishberg DP, et al. Interobserver and intraobserver variability in evaluating vascular invasion in hepatocellular carcinoma. J Gastroenterol Hepatol 2010;25:1556-1561.

25. Mittal S, Kanwal F, Ying J, et al. Effectiveness of surveillance for hepatocellular carcinoma in clinical practice: a United States cohort. J Hepatol 2016;65:1148-1154.

26. Santi V, Trevisani F, Gramenzi A, et al. Semiannual surveillance is superior to annual surveillance for the detection of early hepatocellular carcinoma and patient survival. J Hepatol 2010;53:291297.

27. van Meer S, de Man RA, Coenraad MJ, et al. Surveillance for hepatocellular carcinoma is associated with increased survival: results from a large cohort in the Netherlands. J Hepatol 2015;63:1156-1163.

28. Sangiovanni A, Del Ninno E, Fasani P, et al. Increased survival of cirrhotic patients with a hepatocellular carcinoma detected during surveillance. Gastroenterology 2004;126:1005-1014. 